\title{
Maximal regularity for second order non-autonomous Cauchy problems
}

\author{
by \\ Charles J. K. Batty (Oxford), Ralph Chill (Metz) \\ and SACHI Srivastava (Bangalore and Delhi) \\ Dedicated to J. Kisyński on the occasion of his 75th birthday
}

\begin{abstract}
We consider some non-autonomous second order Cauchy problems of the form

$$
\ddot{u}+B(t) \dot{u}+A(t) u=f \quad(t \in[0, T]), \quad u(0)=\dot{u}(0)=0 .
$$
\end{abstract}

We assume that the first order problem

$$
\dot{u}+B(t) u=f \quad(t \in[0, T]), \quad u(0)=0,
$$

has $L^{p}$-maximal regularity. Then we establish $L^{p}$-maximal regularity of the second order problem in situations when the domains of $B\left(t_{1}\right)$ and $A\left(t_{2}\right)$ always coincide, or when $A(t)=\kappa B(t)$.

1. Introduction. We prove maximal regularity results for some special cases of the non-autonomous second order Cauchy problem

$$
\ddot{u}+B(t) \dot{u}+A(t) u=f \quad(t \in[0, T]), \quad u(0)=\dot{u}(0)=0 .
$$

Here, $B(t)$ and $A(t)$ are (usually unbounded, and not necessarily closed) linear operators on a Banach space $X$.

The terminology of $L^{p}$-maximal regularity for the (autonomous) second order Cauchy problem was introduced by Chill and Srivastava [5], but there are earlier articles in which $L^{p}$-maximal regularity results have been proved. The notion of $L^{p}$-maximal regularity for the second order problem generalises in a suggestive way the notion of $L^{p}$-maximal regularity for the first

2000 Mathematics Subject Classification: Primary 47E05; Secondary 34G10, 35B65, 47D09.

Key words and phrases: maximal regularity, non-autonomous, second order Cauchy problem.

Part for this research was carried out with the support of the Marie Curie "Transfer of Knowledge" programme, "TODEQ". We are very grateful for the financial support from the programme and also for hospitality from Nicolaus Copernicus University of Torun. 
order problem

$$
\dot{u}+B(t) u=f \quad(t \in[0, T]), \quad u(0)=0,
$$

which in turn goes back to the notion of maximal regularity of the sum of two closed operators on a Banach space by Da Prato and Grisvard [8].

Recall that the first order problem (1.2) has $L^{p}$-maximal regularity if, for each $f \in L^{p}(0, T ; X)$, the problem (1.2) admits a unique strong solution $u \in W^{1, p}(0, T ; X)$ such that $u(t) \in D(B(t))$ for almost every $t$ and such that the function $t \mapsto B(t) u(t)$ belongs to $L^{p}(0, T ; X)$ and depends continuously on $f$ with respect to the norm of $L^{p}(0, T ; X)$.

Similarly, the second order problem (1.1) has $L^{p}$-maximal regularity if, for each $f \in L^{p}(0, T ; X)$, the problem (1.1) admits a unique strong solution $u \in W^{2, p}(0, T ; X)$ such that $u(t) \in D(A(t))$ and $\dot{u}(t) \in D(B(t))$ for almost every $t$ and such that the functions $t \mapsto B(t) \dot{u}(t)$ and $t \mapsto A(t) u(t)$ belong to $L^{p}(0, T ; X)$ and depend continuously on $f$ with respect to the norm of $L^{p}(0, T ; X)$. This definition corresponds to maximal regularity of the sum of three operators. However, we shall vary this definition slightly at times.

There are by now only a few maximal regularity results for second order problems. We mention results by J.-L. Lions [9, Chapter XVIII, Section 5], Favini [12], Cannarsa, Da Prato and Zolésio [4] and Arendt, Chill, Fornaro and Poupaud [3, Section 5]. For some results for autonomous problems, see $[5]$.

Maximal regularity results for the non-autonomous first order problem have been proved by J.-L. Lions [9, Chapter XVIII, Section 3], Tanabe [23, Section 5.5], Da Prato and Grisvard [8], Di Blasio [10], [11], Monniaux and Prüss [18], Hieber and Monniaux [13], [14], Prüss and Schnaubelt [20], Amann [2], Portal and Štrkalj [19], Prüss and Simonett [21], Arendt et al. [3], and others.

In this article, we shall assume $L^{p}$-maximal regularity of the first order problem (1.2) in order to establish $L^{p}$-maximal regularity of the second order problem (1.1) in two types of case. Actually, we shall show that this assumption is even necessary in these two cases.

The first case, which we consider in Section 3, is when the domains of $B(t)$ are constant in time and they coincide with the domains of $A(t)$, although the operators $A(t)$ are not necessarily required to be closed on that domain. Maximal regularity of second order problems of this type has previously been considered in [9, Chapter XVIII, Section 5] and [3, Section 5], but our approach, by means of partitions into small subintervals, requires weaker assumptions on the coefficients.

The second case, considered in Section 4, is when $A(t)=\kappa B(t)$, possibly with some additional terms which are relatively small. We shall approach this case by means of factorisations of operators and perturbation methods. 
A problem of this type has previously been considered in Hilbert spaces [4] by other methods. Note that, in the autonomous case, $L^{p}$-maximal regularity has been established in [5] for $A=\kappa B^{\alpha}$ for $1 \leq \alpha<2$, if $X$ is a UMDspace and $B$ has $R$-bounded $H^{\infty}$-functional calculus on a sector of angle less than $\pi / 2$.

We shall illustrate our results in terms of the following specific evolution equation:

$$
\begin{array}{ll}
u_{t t}+\mathcal{B}(t, x, \partial) u_{t}+\mathcal{A}(t, x, \partial) u=f & \text { in }(0, T) \times \Omega, \\
\mathcal{C}_{1}(t, x, \partial) u_{t}+\mathcal{C}_{0}(t, x, \partial) u=0 & \text { in }(0, T) \times \partial \Omega, \\
u(0, x)=u_{0}(x), & \\
u_{t}(0, x)=u_{1}(x), &
\end{array}
$$

where $\Omega$ is a bounded domain in $\mathbb{R}^{n}$, with Lipschitz boundary $\partial \Omega$ and outer normal $\nu$, and

$$
\begin{aligned}
\mathcal{B}(t, x, \partial) & =-\sum_{i, j} \partial_{i}\left(b_{i j}(t, x) \partial_{j}\right)+\sum_{i} b_{i}(t, x) \partial_{i}+b(t, x), \\
\mathcal{A}(t, x, \partial) & =-\sum_{i, j} \partial_{i}\left(a_{i j}(t, x) \partial_{j}\right)+\sum_{i} a_{i}(t, x) \partial_{i}+a(t, x), \\
\mathcal{C}_{1}(t, x, \partial) & =\sum_{i, j} b_{i j}(t, x) \nu_{i}(x) \partial_{j}+c_{1}(t, x), \\
\mathcal{C}_{0}(t, x, \partial) & =\sum_{i, j} a_{i j}(t, x) \nu_{i}(x) \partial_{j}+c_{0}(t, x) .
\end{aligned}
$$

The precise assumptions on $\Omega$, the coefficients and the initial conditions will be given in each example. In fact, under rather weak regularity conditions in time and a uniform ellipticity condition on the coefficients $\left(b_{i j}\right)$, we will prove maximal regularity results in various function spaces.

2. Definition of $L^{p}$-maximal regularity. To address the question of maximal regularity from an abstract point of view, we consider the derivative operator $\mathcal{D}$ on $L^{p}(0, T ; X)$ defined by

$$
\begin{aligned}
D(\mathcal{D}) & =W_{0}^{1, p}(0, T ; X)=\left\{u \in W^{1, p}(0, T ; X): u(0)=0\right\}, \\
\mathcal{D} u & =\dot{u},
\end{aligned}
$$

and multiplication operators $\mathcal{B}, \mathcal{A}$, etc. on $L^{p}(0, T ; X)$ defined by

$$
\begin{aligned}
D(\mathcal{B}) & =\left\{u \in L^{p}(0, T ; X): u(t) \in D(B(t)) \text { a.e., } B(\cdot) u(\cdot) \in L^{p}(0, T ; X)\right\}, \\
(\mathcal{B} u)(t) & =B(t) u(t) \quad(t \in[0, T]),
\end{aligned}
$$

and similarly for $\mathcal{A}$. We will generally adopt the usual conventions for operators $A$ and $B$ that $D(A+B)=D(A) \cap D(B)$ and $D(A B)=$ $\{x \in D(B): B x \in D(A)\}$. 
The first order problem (1.2) is said to have $L^{p}$-maximal regularity if the sum $\mathcal{D}+\mathcal{B}: D(\mathcal{D}) \cap D(\mathcal{B}) \rightarrow L^{p}(0, T ; X)$ is bijective and closed as an operator on $L^{p}(0, T ; X)$. It is then automatic (by the closed graph theorem) that $\mathcal{D}(\mathcal{D}+\mathcal{B})^{-1}$ and $\mathcal{B}(\mathcal{D}+\mathcal{B})^{-1}$ are bounded operators on $L^{p}(0, T ; X)$. Note that this definition is equivalent to the definition we gave in the Introduction.

Similarly, the second order problem (1.1) is said to have $L^{p}$-maximal regularity if the operator $\mathcal{L}:=\mathcal{D}^{2}+\mathcal{B D}+\mathcal{A}$ with natural domain

$$
D(\mathcal{L}):=\left\{u \in W_{0}^{2, p}(0, T ; X) \cap D(\mathcal{A}): \dot{u} \in D(\mathcal{B})\right\},
$$

where

$$
W_{0}^{2, p}(0, T ; X)=\left\{u \in W^{2, p}(0, T ; X): u(0)=\dot{u}(0)=0\right\},
$$

is bijective and $\mathcal{L}^{-1}$ is a bounded operator on $L^{p}(0, T ; X)$. Equivalently, the second order problem (1.1) has $L^{p}$-maximal regularity if $\mathcal{L}$ is bijective from $D(\mathcal{L})$ to $L^{p}(0, T ; X)$ and closed in $L^{p}(0, T ; X)$. Then $\mathcal{L}^{-1}$ is bounded on $L^{p}(0, T ; X)$, by the closed graph theorem. Indeed, when we say that an operator $\mathcal{L}$ (with domain $D(\mathcal{L})$ ) is invertible on an $L^{p}$-space, it is implicit that $\mathcal{L}^{-1}$ is bounded from the $L^{p}$-space to itself.

We shall study the above kind of maximal regularity in Section 3. In Section 4 we shall study the special case when $A(t)=\kappa B(t)$. Then the second order Cauchy problem can be reformulated as

$$
\ddot{u}+B(t)(\dot{u}+\kappa u)=f \quad(t \in[0, T]), \quad u(0)=\dot{u}(0)=0 .
$$

Writing the second order problem in this form instead of (1.1) seems to be suggested, for example, by the partial differential equation (1.3) and in particular by the boundary condition therein. Also Cannarsa et al. [4] write the second order problem in this form. We say that this second order problem has $L^{p}$-maximal regularity if the operator $\widetilde{\mathcal{L}}:=\mathcal{D}^{2}+\mathcal{B}(\mathcal{D}+\kappa)$ with natural domain

$$
D(\widetilde{\mathcal{L}}):=\left\{u \in W_{0}^{2, p}(0, T ; X): \dot{u}+\kappa u \in D(\mathcal{B})\right\}
$$

is bijective and $\widetilde{\mathcal{L}}^{-1}$ is a bounded operator on $L^{p}(0, T ; X)$. Again, this is equivalent to saying that $\widetilde{\mathcal{L}}$ is bijective from $D(\widetilde{\mathcal{L}})$ to $L^{p}(0, T ; X)$ and it is closed in $L^{p}(0, T ; X)$.

Clearly, in the special case $A=\kappa B$ one has $\mathcal{L} \subseteq \widetilde{\mathcal{L}}$ but the two operators may not coincide in general. However, $\mathcal{L}=\widetilde{\mathcal{L}}$ if each operator $B(t)$ is closed, $D(B(t))$ is independent of $t$ and the graph norms on $D(B(t))$ for different $t$ are uniformly equivalent. In fact, let $Y$ be the common Banach space $D(B(t))$. If $u \in D(\widetilde{\mathcal{L}})$ then we may let $v=\dot{u}+\kappa u \in D(\mathcal{B})=L^{p}(0, T ; Y)$. Then

$$
u(t)=\left((\mathcal{D}+\kappa)^{-1} v\right)(t)=\int_{0}^{t} e^{-\kappa(t-s)} v(s) d s \in Y=D(B(t)) \quad \text { a.e. }
$$


Hence $\dot{u}(t)=v(t)-\kappa u(t) \in D(B(t))$ a.e., so $u \in D(\mathcal{L})$. Thus $\mathcal{L}=\widetilde{\mathcal{L}}$ in this case.

Throughout the paper, we will take $T>0$ and $p \in(1, \infty)$ to be fixed. For autonomous Cauchy problems, $L^{p}$-maximal regularity is independent of $T$ and $p$ in this range (see [6] for the second order case). However, $L^{p}$-maximal regularity for the non-autonomous problems (1.2) and (1.1) depends not only on $T$ but also on $p \in(1, \infty)$, in general. This is true even for ordinary differential equations. For example, let

$$
b(t)=\sum_{k=1}^{\infty} c_{k}\left|t-t_{k}\right|^{-1 / p}
$$

for some dense sequence $\left(t_{k}\right)$ in $(0,1)$ and some $c_{k}>0$ with $\sum_{k=1}^{\infty} c_{k}<\infty$. Then $b \in L^{q}(0,1)$ whenever $1<q<p$, and the problem

$$
\dot{u}+b(t) u=f \quad(t \in[0,1]), \quad u(0)=0,
$$

is easily seen to have $L^{q}$-maximal regularity. On the other hand, when $\mathcal{B}$ is considered as an operator on $L^{p}(0,1)$, then $W_{0}^{1, p}(0,1) \cap D(\mathcal{B})=\{0\}$, so $\mathcal{D}+\mathcal{B}$ cannot be invertible on $L^{p}(0,1)$.

Similarly, if $b(t)=b$ is constant but $a(t)=\sum_{k=1}^{\infty} c_{k}\left|t-t_{k}\right|^{-1 / p}$, then the problem

$$
\ddot{u}+b \dot{u}+a(t) u=f \quad(t \in[0,1]), \quad u(0)=\dot{u}(0)=0,
$$

cannot have $L^{p}$-maximal regularity, but it does have $L^{q}$-maximal regularity for $1<q<p$.

We shall also use the terminology of this section, adapted in an obvious way, when $[0, T]$ is replaced by another interval $[a, b]$.

3. The case of constant domains. In this section, we consider the case when each operator $B(t)$ is closed, $D(B(t))=: Y$ is independent of $t \in[0, T]$, and the graph norms are uniformly equivalent. This implies that $D(\mathcal{B})=L^{p}(0, T ; Y)$ (the space $Y$ is equipped with any of the equivalent graph norms). We also assume that $D(A(t))=Y$ and $A(t) \in \mathcal{L}(Y, X)$ for every $t \in[0, T]$, but the operators $A(t)$ need not be closed as operators on $X$. If $A$ is strongly measurable and if $\|A(\cdot)\|_{\mathcal{L}(Y, X)}$ is dominated by an $L^{p}$ function, as we will assume in the following theorem, then

$$
D\left(\mathcal{D}^{2}+\mathcal{B D}+\mathcal{A}\right)=W_{0}^{2, p}(0, T ; X) \cap W^{1, p}(0, T ; Y) .
$$

The following theorem gives a characterisation of unique solvability of the second order problem (1.1) in this domain.

Theorem 3.1. Assume that $B, A:[0, T] \rightarrow \mathcal{L}(Y, X)$ are strongly measurable, and there exists $h \in L^{p}(0, T)$ such that $\|A(t)\|_{\mathcal{L}(Y, X)} \leq h(t)$ for almost every $t$. Then: 
(a) If the first order Cauchy problem

$$
\dot{u}+B(t) u=f \quad(t \in[a, b]), \quad u(a)=0,
$$

has $L^{p}$-maximal regularity for each subinterval $(a, b)$ of $(0, T)$, then the second order problem (1.1) has $L^{p}$-maximal regularity.

(b) If the second order problem

$$
\ddot{u}+B(t) \dot{u}+A(t) u=f \quad(t \in[a, b]), \quad u(a)=\dot{u}(a)=0,
$$

has $L^{p}$-maximal regularity for each subinterval $(a, b)$ of $(0, T)$, then the first order problem (1.2) has $L^{p}$-maximal regularity.

Proof. (a) We have to prove that the operator $\mathcal{L}=\mathcal{D}^{2}+\mathcal{B D}+\mathcal{A}$ with natural domain

$$
D(\mathcal{L})=W_{0}^{2, p}(0, T ; X) \cap W^{1, p}(0, T ; Y)
$$

is invertible on $L^{p}(0, T ; X)$. By assumption, the operators $\mathcal{D}+\mathcal{B}$, with domain $W_{0}^{1, p}(a, b ; X) \cap L^{p}(a, b ; Y)$, are invertible on $L^{p}(a, b ; X)$ for each subinterval $(a, b)$ of $(0, T)$, and $(\mathcal{D}+\mathcal{B})^{-1}: L^{p}(0, T ; X) \rightarrow L^{p}(0, T ; Y)$ is bounded with norm $M$ (say) by the closed graph theorem. The operator $\mathcal{D}$ is invertible on $L^{p}(a, b ; X)$. Hence the operator $\mathcal{D}^{2}+\mathcal{B D}=(\mathcal{D}+\mathcal{B}) \mathcal{D}$, with domain $W_{0}^{2, p}(a, b ; X) \cap W^{1, p}(a, b ; Y)$, is invertible on $L^{p}(a, b ; X)$.

Let $f \in L^{p}(a, b)$ and extend $f$ by 0 to $[0, T]$. Let $u$ be the solution of $(1.2)$ on $[0, T]$. By uniqueness of the solution on $[0, a],\left.u\right|_{[0, a]}=0$. Hence $\left.u\right|_{[a, b]}$ is the unique solution of $(3.1)$ on $[a, b]$. It follows that the norm of $(\mathcal{D}+\mathcal{B})^{-1}$ as an operator from $L^{p}(a, b ; X)$ to $L^{p}(a, b ; Y)$ is at most $M$. The Volterra operator $\mathcal{D}^{-1}: L^{p}(a, b ; Y) \rightarrow L^{\infty}(a, b ; Y)$ has norm $(b-a)^{1 / p^{\prime}}$ and $\mathcal{A}: L^{\infty}(a, b ; Y) \rightarrow L^{p}(a, b ; X)$ has norm at most $\|h\|_{L^{p}(a, b)}$. If $\|h\|_{L^{p}(a, b)}<$ $M^{-1}(b-a)^{-1 / p^{\prime}}$ then the operator $I+\mathcal{A}\left(\mathcal{D}^{2}+\mathcal{B D}\right)^{-1}$ is invertible on $L^{p}(a, b ; X)$. It follows that the operator

$$
\mathcal{D}^{2}+\mathcal{B D}+\mathcal{A}=\left(I+\mathcal{A}\left(\mathcal{D}^{2}+\mathcal{B D}\right)^{-1}\right)\left(\mathcal{D}^{2}+\mathcal{B D}\right),
$$

with domain $W_{0}^{2, p}(a, b ; X) \cap W^{1, p}(a, b ; Y)$, is invertible on $L^{p}(a, b ; X)$.

If $\|h\|_{L^{p}(0, T)}<M^{-1} T^{-1 / p^{\prime}}$, the result is immediate. For the general case, we will subdivide the interval $(0, T)$ into small subintervals on which this special case can be applied and we will build the solutions iteratively.

Choose a partition $0=\tau_{0}<\tau_{1}<\cdots<\tau_{n}=T$ such that $\int_{\tau_{i}}^{\tau_{i+1}} h(t)^{p} d t<$ $M^{-p} T^{-(p-1)}$ for every $i=0, \ldots, n-1$. Such a partition clearly exists. Then $\mathcal{D}^{2}+\mathcal{B D}+\mathcal{A}$, with domain $W_{0}^{2, p}\left(\tau_{i}, \tau_{i+1} ; X\right) \cap W^{1, p}\left(\tau_{i}, \tau_{i+1} ; Y\right)$, is invertible on $L^{p}\left(\tau_{i}, \tau_{i+1} ; X\right)$. This is equivalent to saying that for each $f \in L^{p}\left(\tau_{i}, \tau_{i+1} ; X\right)$ there exists a unique function $u \in W^{2, p}\left(\tau_{i}, \tau_{i+1} ; X\right) \cap$ $W^{1, p}\left(\tau_{i}, \tau_{i+1} ; Y\right)$ which is a solution of

$$
\ddot{u}+B(t) \dot{u}+A(t) u=f \quad\left(t \in\left[\tau_{i}, \tau_{i+1}\right]\right), \quad u\left(\tau_{i}\right)=\dot{u}\left(\tau_{i}\right)=0,
$$


and there exist constants $C_{i}$ such that

$$
\|\ddot{u}\|_{L^{p}\left(\tau_{i}, \tau_{i+1} ; X\right)}+\|\dot{u}\|_{W^{1, p}\left(\tau_{i}, \tau_{i+1} ; Y\right)} \leq C_{i}\|f\|_{L^{p}\left(\tau_{i}, \tau_{i+1} ; X\right)} .
$$

Consider the trace space

$$
\operatorname{Tr}_{p}:=\left\{(u(0), \dot{u}(0)): u \in W^{2, p}(0,1 ; X) \cap W^{1, p}(0,1 ; Y)\right\},
$$

with

$$
\begin{aligned}
& \left\|\left(u_{0}, u_{1}\right)\right\|_{\operatorname{Tr}_{p}}=\inf \left\{\|u\|_{W^{2, p}(0,1 ; X)}+\|u\|_{W^{1, p}(0,1 ; Y)}:\right. \\
& \left.\quad u \in W^{2, p}(0,1 ; X) \cap W^{1, p}(0,1 ; Y), u(0)=u_{0}, u(1)=u_{1}\right\} .
\end{aligned}
$$

This space depends only on the spaces $X$ and $Y$, and not on the choice of the interval $(0,1)$ in its definition. In fact, by [5, Lemma 6.3], for every $i=0, \ldots, n-1$ we have

$$
\begin{aligned}
\operatorname{Tr}_{p} & =\left\{\left(u\left(\tau_{i}\right), \dot{u}\left(\tau_{i}\right)\right): u \in W^{2, p}\left(\tau_{i}, \tau_{i+1} ; X\right) \cap W^{1, p}\left(\tau_{i}, \tau_{i+1} ; Y\right)\right\} \\
& =\left\{\left(u\left(\tau_{i+1}\right), \dot{u}\left(\tau_{i+1}\right)\right): u \in W^{2, p}\left(\tau_{i}, \tau_{i+1} ; X\right) \cap W^{1, p}\left(\tau_{i}, \tau_{i+1} ; Y\right)\right\},
\end{aligned}
$$

with the trace norms being equivalent.

We show that for every $\left(u_{0}, u_{1}\right) \in \operatorname{Tr}_{p}$ the inhomogeneous initial value problem

$$
\ddot{u}+B(t) \dot{u}+A(t) u=f \quad\left(t \in\left[\tau_{i}, \tau_{i+1}\right]\right), \quad u\left(\tau_{i}\right)=u_{0}, \dot{u}\left(\tau_{i}\right)=u_{1},
$$

admits a unique solution $u \in W^{2, p}\left(\tau_{i}, \tau_{i+1} ; X\right) \cap W^{1, p}\left(\tau_{i}, \tau_{i+1} ; Y\right)$, and

$$
\begin{aligned}
\|u\|_{W^{2, p}\left(\tau_{i}, \tau_{i+1} ; X\right)}+\|u\|_{W^{1, p}\left(\tau_{i}, \tau_{i+1} ; Y\right)} & \\
& \leq C_{i}^{\prime}\left(\left\|\left(u_{0}, u_{1}\right)\right\|_{\operatorname{Tr}}+\|f\|_{L^{p}\left(\tau_{i}, \tau_{i+1} ; X\right)}\right) .
\end{aligned}
$$

In fact, let $v \in W^{2, p}\left(\tau_{i}, \tau_{i+1} ; X\right) \cap W^{1, p}\left(\tau_{i}, \tau_{i+1} ; Y\right)$ be such that $v\left(\tau_{i}\right)=u_{0}$ and $\dot{v}\left(\tau_{i}\right)=u_{1}$ and with minimal norm (up to a factor of 2 ). Then solve the inhomogeneous problem (3.2) with $f$ replaced by $\ddot{v}+B(t) \dot{v}+A(t) v+f$, call the solution $w$ and put $u:=v-w$. Then $u$ is a solution of (3.4), and (3.5) holds. Uniqueness of the solution follows from unique solvability of (3.2) and linearity.

Finally, let $f \in L^{p}(0, T ; X)$. Solve first the inhomogeneous problem (3.2) for $i=0$ (i.e., on the interval $\left[\tau_{0}, \tau_{1}\right]$ ) and call the solution $u^{0}$. Then, for $i=1, \ldots, n-1$, we solve iteratively the problem (3.4) with $\left(u_{0}, u_{1}\right)=$ $\left(u^{i-1}\left(\tau_{i}\right), \dot{u}^{i-1}\left(\tau_{i}\right)\right)$ and we call the solution $u^{i}$. These solutions exist and are unique by unique solvability of the problems (3.2) and (3.4) and by linearity. For every $t \in[0, T]$ we put

$$
u(t):=u^{i}(t) \quad \text { if } t \in\left[\tau_{i}, \tau_{i+1}\right] .
$$

Then, by construction, $u \in W_{0}^{2, p}(0, T ; X) \cap W^{1, p}(0, T ; Y)$ is a solution of (1.1). It is easy to verify that this solution must be unique (by showing iteratively that every other solution coincides with $u$ on the intervals 
$\left.\left[\tau_{i}, \tau_{i+1}\right]\right)$ and to show iteratively that $\|u\|_{L^{p}(0, T ; X)} \leq C\|f\|_{L^{p}(0, T ; X)}$. Hence, the problem (1.1) has $L^{p}$-maximal regularity.

(b) By assumption, the operator $\mathcal{D}^{2}+\mathcal{B D}+\mathcal{A}$, with domain $W_{0}^{2, p}(a, b ; X)$ $\cap W^{1, p}(a, b ; Y)$, is invertible on $L^{p}(a, b ; X)$ for each subinterval $(a, b)$ of $(0, T)$. As in the proof of $(a)$, the norm of $\left(\mathcal{D}^{2}+\mathcal{B D}+\mathcal{A}\right)^{-1}$ from $L^{p}(a, b ; X)$ to $W^{1, p}(a, b ; Y)$ is bounded by a constant $M_{1}$ independent of the interval $(a, b)$. Since the norm of the embedding of $W_{0}^{1, p}(a, b ; Y)$ in $L^{\infty}(a, b ; Y)$ is bounded by a constant $M_{2}$ independent of the interval $(a, b)$, it follows that $I-\mathcal{A}\left(\mathcal{D}^{2}+\mathcal{B D}+\mathcal{A}\right)^{-1}$ is invertible on $L^{p}(a, b ; X)$ if $\|h\|_{L^{p}(a, b: X)}<\left(M_{1} M_{2}\right)^{-1}$. Then

$$
(\mathcal{D}+\mathcal{B}) \mathcal{D}=\left(I-\mathcal{A}\left(\mathcal{D}^{2}+\mathcal{B D}+\mathcal{A}\right)^{-1}\right)\left(\mathcal{D}^{2}+\mathcal{B D}+\mathcal{A}\right),
$$

is an isomorphism of $W_{0}^{2, p}(a, b ; X) \cap W^{1, p}(a, b ; Y)$ onto $L^{p}(a, b ; X)$. Hence $\mathcal{D}+\mathcal{B}$ is an isomorphism of $W_{0}^{1, p}(a, b ; X) \cap L^{p}(a, b ; Y)$ onto $L^{p}(a, b ; X)$, so $(3.1)$ has $L^{p}$-maximal regularity when $(a, b)$ is sufficiently small.

Now the proof of (b) proceeds in a very similar way to (a), using the first order trace space

$$
\operatorname{Tr}_{p}^{1}:=\left\{u(0): u \in W^{1, p}(0,1 ; X) \cap L^{p}(0,1 ; Y)\right\},
$$

with

$$
\begin{aligned}
& \left\|u_{0}\right\|_{\operatorname{Tr}_{p}^{1}}=\inf \left\{\|u\|_{W^{1, p}(0,1 ; X)}+\|u\|_{L^{p}(0,1 ; Y)}:\right. \\
& \left.\quad u \in W^{1, p}(0,1 ; X) \cap L^{p}(0,1 ; Y), u(0)=u_{0}\right\} .
\end{aligned}
$$

Under the stronger assumption that $A:[0, T] \rightarrow \mathcal{L}(Y, X)$ is strongly measurable and bounded (but assuming only that (1.2) has maximal regularity on $(0, T)$ ), we can give the following rather simple proof of Theorem 3.1(a).

Second proof of Theorem 3.1(a). Let $Z=W_{0}^{1, p}(0, T ; X) \cap L^{p}(0, T ; Y)$, with the norm $\|f\|_{Z}=\|f\|_{W^{1, p}(0, T ; X)}+\|f\|_{L^{p}(0, T ; Y)}$. By the assumption in (a), $(\mathcal{D}+\mathcal{B})^{-1}$ is a bounded operator from $L^{p}(0, T ; X)$ to $Z$. By assumption in this proof, $\mathcal{A}$ is a bounded operator from $Z$ to $L^{p}(0, T ; X)$. Consequently, $(\mathcal{D}+\mathcal{B})^{-1} \mathcal{A}$ is a bounded operator on $Z$.

The part of $\mathcal{D}$ in $Z, \mathcal{D}_{Z}$, has domain $W_{0}^{2, p}(0, T ; X) \cap W^{1, p}(0, T ; Y)$, and $-\mathcal{D}_{Z}$ generates the nilpotent $C_{0}$-semigroup of right shifts on $Z$. Hence the bounded perturbation $-\mathcal{D}_{Z}-(\mathcal{D}+\mathcal{B})^{-1} \mathcal{A}$ generates a $C_{0}$-semigroup of growth bound $-\infty$, and so it is invertible. Thus $\mathcal{D}_{Z}+(\mathcal{D}+\mathcal{B})^{-1} \mathcal{A}$ is an isomorphism of $W_{0}^{2, p}(0, T ; X) \cap W^{1, p}(0, T ; Y)$ onto $Z$. Since $\mathcal{D}+\mathcal{B}$ is an isomorphism of $Z$ onto $L^{p}(0, T ; X)$, the result follows from the factorisation

$$
\mathcal{L}=(\mathcal{D}+\mathcal{B})\left(\mathcal{D}_{Z}+(\mathcal{D}+\mathcal{B})^{-1} \mathcal{A}\right)
$$


In the case of constant domains, there are basically two situations in which the first order problem (1.2) is known to have maximal regularity:

- the variational case considered in [9, Chapter XVIII, Section 3] and [23, Theorem 5.5.1]; in this case the underlying space is a Hilbert space, the operators $B(t)$ are associated with bounded bilinear forms, and $B(t)$ may be only measurable in time;

- the case when the autonomous first order problems $\dot{u}+B\left(t^{\prime}\right) u=f$ have $L^{p}$-maximal regularity for each $t^{\prime} \in[0, T]$ (we say for short that $B\left(t^{\prime}\right)$ has $L^{p}$-maximal regularity for each $t^{\prime}$ ) and the function $B$ is relatively continuous in time (see [3] for this result and the definition of relative continuity, and also [20] and [2] for the case of continuous dependence on time).

These two cases, together with Theorem 3.1, lead to the applications which follow in Corollary 3.2 and Corollary 3.6 respectively.

In the first application of Theorem 3.1 we consider the situation when the underlying Banach space is a Hilbert space and the operators $B(t)$ are associated with bounded bilinear forms.

COROLlary 3.2. Let $V$ and $H$ be real separable Hilbert spaces such that $V \subset H=H^{\prime} \subset V^{\prime}$ with continuous and dense embeddings. Let $A(t), B(t)$ : $V \rightarrow V^{\prime}$ be bounded linear operators which are weakly measurable in time and which satisfy

$$
\begin{aligned}
& \|B(t)\|_{\mathcal{L}\left(V, V^{\prime}\right)} \leq C, \\
& \langle B(t) u, u\rangle_{V^{\prime}, V}+\omega\|u\|_{H}^{2} \geq c\|u\|_{V}^{2} \quad(u \in V), \\
& \|A(\cdot)\|_{\mathcal{L}\left(V, V^{\prime}\right)} \in L^{2}(0, T)
\end{aligned}
$$

for some positive constants $C, \omega$ and $c$. Then the second order problem (1.1) has $L^{2}$-maximal regularity on the domain $W_{0}^{2,2}\left(0, T ; V^{\prime}\right) \cap W^{1,2}(0, T ; V)$ with respect to $X=V^{\prime}$.

Proof. It suffices to note that the first order problem (1.2) has $L^{2}$ maximal regularity by $[23$, Theorem 5.5 .1$]$ (see also [9, Théorème 2, p. 620] for the case of symmetric $A$ and $B$ ) and to apply Theorem 3.1.

REMARK 3.3. In the case of symmetric $A$ and $B$, J.-L. Lions already considered $L^{2}$-maximal regularity of the second order Cauchy problem (1.1) in the variational situation of Corollary 3.2 above. He proved $L^{2}$-maximal regularity under the stronger regularity assumptions that $B$ is weakly continuous and $A$ is weakly continuously differentiable in time $[9$, Théorème 1 , p. 670]. Theorem 3.1 removes these regularity conditions.

EXAMPLE 3.4. We consider the problem (1.3). We assume that $\Omega \subset \mathbb{R}^{n}$ is a domain with Lipschitz boundary. The coefficients satisfy the following 
regularity conditions:

$$
\begin{aligned}
& b_{i j} \in L^{\infty}\left(0, T ; L^{\infty}(\Omega)\right), \\
& b_{i}, b \in L^{\infty}\left(0, T ; L^{\infty}(\Omega)\right), \\
& c_{1} \in L^{\infty}\left(0, T ; L^{\infty}(\partial \Omega)\right) \text { and } c_{1} \geq 0, \\
& a_{i j} \in L^{p}\left(0, T ; L^{\infty}(\Omega)\right), \\
& a_{i}, a \in L^{p}\left(0, T ; L^{\infty}(\Omega)\right), \\
& c_{0} \in L^{p}\left(0, T ; L^{\infty}(\partial \Omega)\right) \text { and } c_{0} \geq 0 .
\end{aligned}
$$

Moreover, the coefficients $b_{i j}$ are assumed to be uniformly elliptic; that is, we assume that there exists a constant $\eta>0$ such that for every $t \in[0, T]$, every $x \in \Omega$, and every $\xi \in \mathbb{R}^{n}$,

$$
\sum_{i, j} b_{i j}(t, x) \xi_{i} \xi_{j} \geq \eta|\xi|^{2} .
$$

We call a function $u \in W^{2,2}\left(0, T ; H^{1}(\Omega)^{\prime}\right) \cap W^{1,2}\left(0, T ; H^{1}(\Omega)\right)$ a weak solution of (1.3) if $u$ satisfies the initial conditions and, for each test function $\varphi \in C_{c}^{1}\left((0, T) ; H^{1}(\Omega)\right)$, the equality

$$
\begin{aligned}
& -\int_{0}^{T} \int_{\Omega} u_{t} \varphi_{t}+\int_{0}^{T} \int_{\Omega} \sum_{i, j}\left(b_{i j} \partial_{j} u_{t} \partial_{i} \varphi+a_{i j} \partial_{j} u \partial_{i} \varphi\right) \\
& +\int_{0}^{T} \int_{\Omega} \sum_{i}\left(b_{i} \partial_{i} u_{t} \varphi+a_{i} \partial_{i} u \varphi\right)+\int_{0}^{T} \int_{\Omega}\left(b u_{t} \varphi+a u \varphi\right) \\
& +\int_{0}^{T} \int_{\partial \Omega}\left(c_{1} u_{t} \varphi+c_{0} u \varphi\right)=\int_{0}^{T}\langle f, \varphi\rangle_{\left(H^{1}\right)^{\prime}, H^{1}}
\end{aligned}
$$

holds (the integrals over the boundary $\partial \Omega$ are understood with respect to the $(n-1)$-dimensional Hausdorff measure). This equality is obtained for regular solutions and coefficients when multiplying equation (1.3) by $\varphi$, integrating over $(0, T) \times \Omega$ and several integrations by parts.

Corollary 3.5. Assume that (3.9)-(3.11) hold with $p=2$. Then for every $f \in L^{2}\left(0, T ; H^{1}(\Omega)^{\prime}\right)$, every $u_{0} \in H^{1}(\Omega)$ and every $u_{1} \in L^{2}(\Omega)$ the problem (1.3) admits a unique weak solution

$$
u \in W^{2,2}\left(0, T ; H^{1}(\Omega)^{\prime}\right) \cap W^{1,2}\left(0, T ; H^{1}(\Omega)\right) .
$$

Proof. We first note that weak solutions are solutions of the abstract second order Cauchy problem

$$
\ddot{u}+B(t) \dot{u}+A(t) u=f \quad(t \in[0, T]), \quad u(0)=u_{0}, \dot{u}(0)=u_{0},
$$


in $X=H^{1}(\Omega)^{\prime}$, if the operators $B(t), A(t): H^{1}(\Omega) \rightarrow H^{1}(\Omega)^{\prime}$ are given by

$$
\langle B(t) u, \varphi\rangle_{\left(H^{1}\right)^{\prime}, H^{1}}=\int_{\Omega} \sum_{i, j} b_{i j} \partial_{j} u \partial_{i} \varphi+\int_{\Omega} \sum_{i} b_{i} \partial_{i} u \varphi+\int_{\Omega} b u \varphi+\int_{\partial \Omega} c_{1} u \varphi
$$

and

$$
\langle A(t) u, \varphi\rangle_{\left(H^{1}\right)^{\prime}, H^{1}}=\int_{\Omega} \sum_{i, j} a_{i j} \partial_{j} u \partial_{i} \varphi+\int_{\Omega} \sum_{i} a_{i} \partial_{i} u \varphi+\int_{\Omega} a u \varphi+\int_{\partial \Omega} c_{0} u \varphi
$$

for $u, \varphi \in H^{1}(\Omega)$. Conversely, if $u \in W^{2,2}\left(0, T ; H^{1}(\Omega)^{\prime}\right) \cap W^{1,2}\left(0, T ; H^{1}(\Omega)\right)$ is a solution of (3.14), then $u$ is a weak solution of (1.3); this can be seen from multiplying (3.14) by $\varphi$ with respect to the duality $\langle\cdot, \cdot\rangle_{\left(H^{1}\right)^{\prime}, H^{1}}$, integrating over $(0, T)$ and using the definition of the operators $B(t)$ and $A(t)$.

By the regularity and boundedness conditions (3.6), (3.7), (3.9), (3.10), the operators $B(t)$ and $A(t)$ are weakly measurable in time, and they satisfy the boundedness conditions of Corollary 2.3 (with $V=H^{1}$ ). The ellipticity condition (3.12) and the positivity condition in (3.8) and (3.11) imply that the operators $B(t)$ satisfy the ellipticity condition of Corollary 2.3 (with $\left.H=L^{2}\right)$. Hence, by Corollary 2.3, problem (1.1) has $L^{2}$-maximal regularity. This ensures existence and uniqueness of solutions for the inhomogeneous problem if $u_{0}=u_{1}=0$. In much the same way as for the initial value problem (3.4), one shows that then (3.14) also admits a solution if $\left(u_{0}, u_{1}\right)$ belongs to the trace space $\operatorname{Tr}_{2}$ defined in (3.3) (with $X=H^{1}(\Omega)^{\prime}$ and $Y=H^{1}(\Omega)$ ). By [5, Lemma 6.2] and [9, Remarque 8, p. 631], $\operatorname{Tr}_{2}=H^{1}(\Omega) \times L^{2}(\Omega)$, and the proof is complete.

We now give applications of Theorem 3.1 in the other situation, when (1.2) has maximal regularity due to relative continuity of $B$ with respect to the embedding of the common domain $Y$ in $X$. For the definition of relative continuity of operator-valued functions used in the following corollary, see [3, Definition 2.5], and note that every continuous function is relatively continuous. The following result generalises [3, Theorem 5.6] by weakening the assumptions on regularity of $A$.

Corollary 3.6. Assume that the function $B:[0, T] \rightarrow \mathcal{L}(Y, X)$ is strongly measurable and relatively continuous, and assume that each $B(t)$ has $L^{p}$-maximal regularity for the first order autonomous problem. Assume that $A:[0, T] \rightarrow \mathcal{L}(Y, X)$ is strongly measurable and that $\|A(\cdot)\|$ is dominated by a function in $L^{p}(0, T)$. Then the second order problem (1.1) has $L^{p}$-maximal regularity.

Proof. The assumptions on $B$ (constant domain, maximal regularity for each $B(t)$ and relative continuity) imply that the first order problem (1.2) has $L^{p}$-maximal regularity by [3, Theorem 2.7]. Thus the claim follows from our Theorem 3.1 . 
EXAMPLE 3.7. We consider again the problem (1.3). Let $\Omega \subset \mathbb{R}^{n}$ be a bounded domain with boundary $\partial \Omega$ of class $C^{2}$, and fix $p \in(1, \infty)$. In this example, the coefficients satisfy the following regularity conditions:

$$
\begin{aligned}
& b_{i j} \in C\left([0, T] ; C^{1}(\Omega)\right), \\
& a_{i j} \in L^{p}\left(0, T ; W^{1, \infty}(\Omega)\right) .
\end{aligned}
$$

For the lower order coefficients $b_{i}, b, a_{i}$ and $a$ we assume (3.7) and (3.10), as in Example 3.4.

We also assume that the boundary conditions do not depend on time, and that the two boundary operators $\mathcal{C}_{1}$ and $\mathcal{C}_{0}$ coincide. More precisely, we assume that there exist functions

$$
d_{j}, c \in C^{1}(\partial \Omega)
$$

such that for every $(t, x) \in[0, T] \times \partial \Omega$,

$$
\begin{gathered}
d_{j}(x)=\sum_{i} b_{i j}(t, x) \nu_{i}(x)=\sum_{i} a_{i j}(t, x) \nu_{i}(x), \\
c(x)=c_{1}(t, x)=c_{0}(t, x) \quad \text { and } \quad c \geq 0 .
\end{gathered}
$$

Finally, as in Example 3.4, we assume that the ellipticity condition (3.12) on the coefficients $b_{i j}$ holds.

For $q \in(1, \infty)$ we consider the space

$$
Y_{q}:=\left\{u \in W^{2, q}(\Omega): \sum_{j} d_{j} \partial_{j} u+c u=0 \text { on } \partial \Omega\right\},
$$

which is a Banach space when it is equipped with the $W^{2, q}$-norm.

Corollary 3.8. Let $p, q \in(1, \infty)$. Then, for each $f \in L^{p}\left(0, T ; L^{q}(\Omega)\right)$, each $u_{0} \in Y_{q}$ and each $u_{1} \in\left(L^{q}(\Omega), Y_{q}\right)_{1 / p^{\prime}, p}$ (the real interpolation space), there exists a unique function

$$
u \in W^{2, p}\left(0, T ; L^{q}(\Omega)\right) \cap W^{1, p}\left(0, T ; Y_{q}\right),
$$

which is a solution of the problem (1.3) in the following sense: the function $u$ satisfies the differential equation in (1.3) almost everywhere (the derivatives all being understood in the Sobolev sense), the initial conditions are satisfied, as well as the boundary conditions

$$
\sum_{j} d_{j} \partial_{j} u_{t}+c u_{t}=\sum_{j} d_{j} \partial_{j} u+c u=0 .
$$

In particular, all terms on the left-hand side of the differential equation in (1.3) belong to $L^{p}\left(0, T ; L^{q}(\Omega)\right)$.

Proof. We consider again the operators $B(t): H^{1}(\Omega) \rightarrow H^{1}(\Omega)^{\prime}$ given by (3.15). It is well known that each $B(t)$, when restricted to $H^{1}(\Omega) \cap L^{q}(\Omega)$, extends in a unique way to a negative generator of a $C_{0}$-semigroup on $L^{q}(\Omega)$ [17, Section 3.1.1]. This negative generator on $L^{q}(\Omega)$ will be denoted again 
by $B(t)$. By [17, Theorem 3.1.2], we have $D(B(t))=Y_{q}$, where $Y_{q}$ is as in $(3.21)$.

Fix $t \in[0, T]$. By [7, Theorem 6.1], the semigroup generated by $-B(t)$ satisfies Gaussian estimates. By [15, Theorem 3.1], the operator $B(t)-\omega_{t}$ has $L^{p}$-maximal regularity for the autonomous problem on the half-line $\mathbb{R}_{+}$, which implies that $B(t)$ has $L^{p}$-maximal regularity for the autonomous problem on the finite interval $[0, T]$.

By the continuity in time of the second order coefficients $b_{i j}$ (condition (3.16)), by condition (3.7), and by following the arguments from the proof of [3, Theorem 4.1], one deduces that the function $B$ is strongly measurable and relatively continuous.

Finally, if we define the operators $A(t): Y_{q} \rightarrow L^{q}(\Omega)$ by

$$
A(t) u=\sum_{i, j} a_{i j} \partial_{i} \partial_{j} u+\sum_{j}\left(\sum_{i} \partial_{i} a_{i j}+a_{j}\right) \partial_{j} u+a u,
$$

then $A(t)$ is bounded for almost every $t \in[0, T]$. Moreover, the function $A$ is strongly measurable. By (3.17) and (3.10), and since $L^{q}(\Omega)$ is separable, $\|A(\cdot)\|$ belongs to $L^{p}(0, T)$. By Corollary 3.6, the problem (1.1) has $L^{p}$ maximal regularity. Solvability for the initial value problem follows as in Example 3.4.

4. The case when $A=\kappa B$. In this section we consider the case when $A(t)=\kappa B(t)$ for some constant $\kappa$ (and in particular, $D(A(t))=D(B(t)))$, but the domain of $B(t)$ may vary with $t$. To be more precise, we consider the problem

$$
\ddot{u}+B(t)(\dot{u}+\kappa u)=f \quad(t \in[0, T]), \quad u(0)=\dot{u}(0)=0,
$$

which slightly differs from the original second order problem (1.1). We remark that second order problems of this type appear in $[4$, Theorems 1.3.2, 2.2.1], and they are also suggested by the type of boundary conditions in our problem (1.3); see also Example 3.4, and Example 4.3 below.

For the problem of $L^{p}$-maximal regularity, we consider the operators $\mathcal{D}$ and $\mathcal{B}$ on $L^{p}(0, T ; X)$ defined by $(2.1)$ and $(2.2)$. Note that the spectrum of $\mathcal{D}$ is empty and

$$
\left((\mathcal{D}+\lambda)^{-1} f\right)(t)=\int_{0}^{t} e^{-\lambda(t-s)} f(s) d s \quad\left(f \in L^{p}(0, T ; X), t \in[0, T]\right) .
$$

For $\lambda \in \mathbb{C}$, let $E_{\lambda}$ be the bounded operator on $L^{p}(0 ; T ; X)$ defined by

$$
\left(E_{\lambda} f\right)(t)=e^{\lambda t} f(t) \text {. }
$$

Then $E_{\lambda}$ is invertible, $E_{\lambda}^{-1}=E_{-\lambda}$ and

$$
E_{-\lambda} \mathcal{B} E_{\lambda}=\mathcal{B}, \quad E_{-\lambda} \mathcal{D} E_{\lambda}=\mathcal{D}+\lambda, \quad E_{-\lambda} \mathcal{D}^{-1} E_{\lambda}=(\mathcal{D}+\lambda)^{-1} .
$$


For technical reasons, we shall assume that the operator $\mathcal{D}+\mathcal{B}$ is closable, its closure has non-empty resolvent set (from which it follows by the similarity relations (4.2) that the resolvent set is $\mathbb{C}$ ) and

$$
\left\|(\overline{\mathcal{D}+\mathcal{B}}+\lambda)^{-1}\right\|=O\left(\lambda^{-1}\right) \quad \text { as } \lambda \rightarrow \infty .
$$

To establish maximal regularity of (4.1), we continue to assume that (1.2) has maximal regularity. There are several results in the literature identifying conditions which are sufficient to ensure that (1.2) has $L^{p}$-maximal regularity or the corresponding property in Hölder spaces, without assuming that the domains $D(B(t))$ are constant. Instead they rely on regularity conditions on $\{B(t)\}$ due to Acquistapace and Terreni [1] or Kato and Tanabe [16], [23]. We mention the following cases:

- $X$ is a Hilbert space and the operators $B(t)$ satisfy the AcquistapaceTerreni conditions [13];

- $X$ is a UMD space and the operators $B(t)$ satisfy the AcquistapaceTerreni conditions and are uniformly $R$-sectorial [22], [19];

- $X$ is a UMD space and the operators $B(t)$ satisfy the Kato-Tanabe conditions and are uniformly $R$-sectorial [21].

In each of these cases, the assumption (4.3) is satisfied, so Theorem 4.1 below shows that (4.1) has maximal regularity. When $X$ is a Hilbert space, a related result has been given by Cannarsa, Da Prato and Zolésio [4, Theorem 1.3.2].

TheOREM 4.1. Assume that (4.3) holds. The second order problem (4.1) has $L^{p}$-maximal regularity if and only if the first order problem (1.2) has $L^{p}$-maximal regularity.

Proof. Assume first that (1.2) has $L^{p}$-maximal regularity, so that $\mathcal{D}+\mathcal{B}$ is closed and invertible. Since $\kappa \mathcal{D}(\mathcal{D}+\kappa)^{-1}$ is bounded, this implies that $\mathcal{D}+\mathcal{B}-\kappa \mathcal{D}(\mathcal{D}+\kappa)^{-1}$ is closed. By our assumption (4.3), there exists $\lambda \in \mathbb{C}$ such that $\lambda>0, \mathcal{D}+\mathcal{B}+\lambda$ is invertible, and $\left\|(\mathcal{D}+\mathcal{B}+\lambda)^{-1}\right\|<1 / 2 \kappa$. Then

$$
\begin{aligned}
\mathcal{D}+\mathcal{B}+\lambda & -\kappa(\mathcal{D}+\lambda)(\mathcal{D}+\lambda+\kappa)^{-1} \\
& =\left(I-\kappa\left(I-\kappa(\mathcal{D}+\lambda+\kappa)^{-1}\right)(\mathcal{D}+\mathcal{B}+\lambda)^{-1}\right)(\mathcal{D}+\mathcal{B}+\lambda)
\end{aligned}
$$

is invertible. By $(4.2), \mathcal{D}+\mathcal{B}-\kappa \mathcal{D}(\mathcal{D}+\kappa)^{-1}$ is invertible. Thus the problem (4.1) has $L^{p}$-maximal regularity.

Conversely, assume that (4.1) has $L^{p}$-maximal regularity, so that $\mathcal{D}+\mathcal{B}$ $-\kappa \mathcal{D}(\mathcal{D}+\kappa)^{-1}$ is invertible. Since $\kappa \mathcal{D}(\mathcal{D}+\kappa)^{-1}$ is bounded, this implies that $\mathcal{D}+\mathcal{B}$ is closed. By our assumption (4.3), $D+\mathcal{B}+\lambda$ is invertible for some $\lambda$, and then $\mathcal{D}+\mathcal{B}$ is invertible by (4.2). Since

$$
\widetilde{\mathcal{L}}=\mathcal{D}^{2}+\mathcal{B}(\mathcal{D}+\kappa)=\left(\mathcal{D}+\mathcal{B}-\kappa \mathcal{D}(\mathcal{D}+\kappa)^{-1}\right)(\mathcal{D}+\kappa),
$$

this implies that (1.2) has $L^{p}$-maximal regularity. 
Now we give a further theorem of this type involving perturbations of the equation (4.1). Given an operator $B$ on $X$ and a Banach space $Y$ such that $D(B) \hookrightarrow Y \hookrightarrow X$, we say that $Y$ is close to $X$ compared to $D(B)$ if, for every $\varepsilon>0$, there exists $c_{\varepsilon}>0$ such that

$$
\|x\|_{Y} \leq \varepsilon\|B x\|_{X}+c_{\varepsilon}\|x\|_{X}
$$

for all $x \in D(B)$, as in [3, p. 12]. Given a family of operators $(B(t))_{t \in[0, T]}$ such that $D(B(t)) \hookrightarrow Y$ for each $t \in[0, T]$, we say that $Y$ is close to $X$ compared to $D(B(t))$, uniformly in $t$, if $c_{\varepsilon}$ can be chosen independently of $t$. This implies that $L^{p}(0, T ; Y)$ is close to $L^{p}(0, T ; X)$ compared to $D(\mathcal{B})$.

TheOREM 4.2. Assume that there is a Banach space $Y$ such that $D(B(t)) \hookrightarrow Y \hookrightarrow X$ and $Y$ is close to $X$ compared to $D(B(t))$ uniformly in $t$. Assume that (4.3) holds and that (1.2) has $L^{p}$-maximal regularity. Let $A_{0}, B_{0}:[0, T] \rightarrow \mathcal{L}(Y, X)$ be bounded and strongly measurable. Then the problem

$$
\begin{aligned}
& \ddot{u}+B(t)(\dot{u}+\kappa u)+B_{0}(t) \dot{u}+A_{0}(t) u=f \quad(t \in[0, T]), \\
& u(0)=\dot{u}(0)=0,
\end{aligned}
$$

has $L^{p}$-maximal regularity.

Proof. By Theorem 4.1,

$$
\widetilde{\mathcal{L}}=\mathcal{D}^{2}+\mathcal{B}(\mathcal{D}+\kappa)=\left(\mathcal{D}+\mathcal{B}-\kappa \mathcal{D}(\mathcal{D}+\kappa)^{-1}\right)(\mathcal{D}+\kappa)
$$

is invertible, and by $(4.2)$ the similar operator $(\mathcal{D}+\lambda)^{2}+\mathcal{B}(\mathcal{D}+\lambda+\kappa)$ is invertible for each $\lambda \in \mathbb{C}$. We have to show that $\mathcal{L}^{\prime}:=\mathcal{D}^{2}+\mathcal{B}(\mathcal{D}+\kappa)+$ $\mathcal{B}_{0} \mathcal{D}+\mathcal{A}_{0}$ is invertible with domain $D(\widetilde{\mathcal{L}})$.

Let $u \in D(\widetilde{\mathcal{L}})$. Then $(\mathcal{D}+\kappa) u \in D(\mathcal{B}) \subseteq L^{p}(0, T ; Y)$, so $u=$ $(\mathcal{D}+\kappa)^{-1}(\mathcal{D}+\kappa) u \in W^{1, p}(0, T ; Y)$. Hence, $u \in D\left(\mathcal{L}^{\prime}\right)$. Thus, $D\left(\mathcal{L}^{\prime}\right)=D(\widetilde{\mathcal{L}})$.

Let $\lambda \in \mathbb{C}$. By $(4.2), \mathcal{L}^{\prime}$ is similar to

$$
\begin{aligned}
& (4.5) \quad(\mathcal{D}+\lambda)^{2}+\mathcal{B}(\mathcal{D}+\lambda+\kappa)+\mathcal{B}_{0}(\mathcal{D}+\lambda)+\mathcal{A}_{0} \\
& =\left(I+\left(\mathcal{B}_{0}(\mathcal{D}+\lambda)+\mathcal{A}_{0}\right)\left((\mathcal{D}+\lambda)^{2}+\mathcal{B}(\mathcal{D}+\lambda+\kappa)\right)^{-1}\right)\left((\mathcal{D}+\lambda)^{2}+\mathcal{B}(\mathcal{D}+\lambda+\kappa)\right) .
\end{aligned}
$$

For any $f \in L^{p}(0, T ; X)$ and $\varepsilon>0$,

$$
\begin{aligned}
&\left\|\mathcal{B}_{0}(\mathcal{D}+\lambda)\left((\mathcal{D}+\lambda)^{2}+\mathcal{B}(\mathcal{D}+\lambda+\kappa)\right)^{-1} f\right\|_{L^{p}(0, T ; X)} \\
& \leq C\left\|(\mathcal{D}+\lambda)\left((\mathcal{D}+\lambda)^{2}+\mathcal{B}(\mathcal{D}+\lambda+\kappa)\right)^{-1} f\right\|_{L^{p}(0, T ; Y)} \\
&= C\left\|(D+\lambda)(\mathcal{D}+\lambda+\kappa)^{-1}\left(\mathcal{D}+\lambda+\mathcal{B}-\kappa(\mathcal{D}+\lambda)(\mathcal{D}+\lambda+\kappa)^{-1}\right)^{-1} f\right\|_{L^{p}(0, T ; Y)} \\
& \leq 2 C\left\|\left(\mathcal{D}+\lambda+\mathcal{B}-\kappa(\mathcal{D}+\lambda)(\mathcal{D}+\lambda+\kappa)^{-1}\right)^{-1} f\right\|_{L^{p}(0, T ; Y)} \\
& \leq 2 C \varepsilon\left\|\mathcal{B}\left(\mathcal{D}+\lambda+\mathcal{B}-\kappa(\mathcal{D}+\lambda)(\mathcal{D}+\lambda+\kappa)^{-1}\right)^{-1} f\right\|_{L^{p}(0, T ; X)} \\
&+2 C c_{\varepsilon}\left\|\left(\mathcal{D}+\lambda+\mathcal{B}-\kappa(\mathcal{D}+\lambda)(\mathcal{D}+\lambda+\kappa)^{-1}\right)^{-1} f\right\|_{L^{p}(0, T ; X)}
\end{aligned}
$$


By assumption (4.3), $\left\|(\mathcal{D}+\lambda+\mathcal{B})^{-1}\right\|_{\mathcal{L}\left(L^{p}(0, T ; X)\right)} \rightarrow 0$ as $\lambda \rightarrow \infty$ and $\left\|\mathcal{B}(\mathcal{D}+\lambda+\mathcal{B})^{-1}\right\|=\left\|\mathcal{B}(\mathcal{D}+\mathcal{B})^{-1}\left(I-\lambda(\mathcal{D}+\lambda+\mathcal{B})^{-1}\right)\right\|_{\mathcal{L}\left(L^{p}(0, T ; X)\right)} \leq M$ for all sufficiently large $\lambda>0$. Hence $\left\|\mathcal{B}\left(\mathcal{D}+\lambda+\mathcal{B}-\kappa(\mathcal{D}+\lambda)(\mathcal{D}+\lambda+\kappa)^{-1}\right)^{-1}\right\|_{\mathcal{L}\left(L^{p}(0, T ; X)\right)}$
$\quad=\left\|\mathcal{B}(\mathcal{D}+\lambda-1+\mathcal{B})^{-1}\left(I+\kappa^{2}(\mathcal{D}+\lambda+\kappa)^{-1}(\mathcal{D}+\lambda-1+\mathcal{B})^{-1}\right)^{-1}\right\|_{\mathcal{L}\left(L^{p}(0, T ; X)\right)}$
$\quad \leq M^{\prime}$ for all sufficiently large $\lambda$, and

$$
\begin{aligned}
& \left\|\left(\mathcal{D}+\lambda+\mathcal{B}-\kappa(\mathcal{D}+\lambda)(\mathcal{D}+\lambda+\kappa)^{-1}\right)^{-1}\right\|_{\mathcal{L}\left(L^{p}(0, T ; X)\right)} \\
& \quad=\left\|(\mathcal{D}+\lambda-1+\mathcal{B})^{-1}\left(I+\kappa^{2}(\mathcal{D}+\lambda+\kappa)^{-1}(\mathcal{D}+\lambda-1+\mathcal{B})^{-1}\right)^{-1}\right\|_{\mathcal{L}\left(L^{p}(0, T ; X)\right)} \\
& \quad \rightarrow 0
\end{aligned}
$$

as $\lambda \rightarrow \infty$. We may choose $\varepsilon>0$ so small that $2 C \varepsilon M^{\prime}<1 / 8$ and then choose $\lambda>0$ large enough so that

$$
2 C c_{\varepsilon}\left\|\left(\mathcal{D}+\lambda+\mathcal{B}-\kappa(\mathcal{D}+\lambda)(\mathcal{D}+\lambda+\kappa)^{-1}\right)^{-1}\right\|_{L^{p}(0, T ; X)}<1 / 8 .
$$

It follows that

$$
\left\|\mathcal{B}_{0}(\mathcal{D}+\lambda)\left((\mathcal{D}+\lambda)^{2}+\mathcal{B}(\mathcal{D}+\lambda+\kappa)\right)^{-1}\right\|_{\mathcal{L}\left(L^{p}(0, T ; X)\right)}<1 / 4 .
$$

Similarly we can arrange that

$$
\left\|\mathcal{A}_{0}\left((\mathcal{D}+\lambda)^{2}+\mathcal{B}(\mathcal{D}+\lambda+\kappa)\right)^{-1}\right\|_{\mathcal{L}\left(L^{p}(0, T ; X)\right)}<1 / 4 .
$$

Then it follows from (4.5) that $(\mathcal{D}+\lambda)^{2}+\mathcal{B}(\mathcal{D}+\lambda+\kappa)+\mathcal{B}_{0} \mathcal{D}+\mathcal{A}_{0}$ is invertible. By $(4.2), \mathcal{L}^{\prime}$ is invertible and the claim is proved.

EXAMPLE 4.3. We consider again the problem (1.3). Let $\Omega \subset \mathbb{R}^{n}$ be a bounded domain with boundary $\partial \Omega$ of class $C^{2}$. We make the following assumptions:

$$
\begin{aligned}
& b_{i j} \in C\left([0, T] ; C^{1}(\bar{\Omega})\right) \cap C^{\mu}([0, T] ; C(\bar{\Omega})), \quad \text { where } 0<\mu \leq 1, \\
& b_{i j}=b_{j i}=a_{i j}=a_{j i}, \\
& c_{1}=c_{0} \in C\left([0, T] ; C^{1}(\partial \Omega)\right) \cap C^{\mu}([0, T] ; C(\partial \Omega)) \quad \text { and } \quad c_{1} \geq 0 .
\end{aligned}
$$

The lower order coefficients $b_{i}, b, a_{i}$ and $a$ satisfy the conditions (3.7) and (3.10) from Example 3.4 with $p=\infty$. We assume in addition the ellipticity condition (3.12) on the coefficients $b_{i j}$.

Corollary 4.4. Let $p, q \in(1, \infty)$ and assume that $u_{0}=u_{1}=0$. Then for every $f \in L^{p}\left(0, T ; L^{q}(\Omega)\right)$ there exists a unique function

$$
u \in W^{2, p}\left(0, T ; L^{q}(\Omega)\right) \cap W^{1, p}\left(0, T ; W^{2, q}(\Omega)\right)
$$

which is a solution of the problem (1.3) in the following sense: the function $u$ satisfies the differential equation in (1.3) almost everywhere (all the derivatives being understood in the Sobolev sense), and the initial and boundary 
conditions are satisfied almost everywhere. In particular, all terms on the left-hand side of the differential equation in (1.3) belong to $L^{p}\left(0, T ; L^{q}(\Omega)\right)$.

Proof. For every $t \in[0, T]$, we consider the operator $B(t)$ on $L^{q}(\Omega)$ defined by

$$
\begin{aligned}
D(B(t)) & :=\left\{u \in W^{2, p}(\Omega): \mathcal{C}^{1}(t, \cdot, \partial) u=0 \text { on } \partial \Omega\right\}, \\
B(t) u & :=-\sum_{i, j} \partial_{i}\left(b_{i j}(t, x) \partial_{j} u\right) .
\end{aligned}
$$

We also define operators $B_{0}(t), A_{0}(t): W^{1, q}(\Omega) \rightarrow L^{q}(\Omega)$ by

$$
B_{0}(t) u:=\sum_{i} b_{i}(t, x) \partial_{i} u+b(t, x) u, \quad A_{0}(t) u:=\sum_{i} a_{i}(t, x) \partial_{i} u+a(t, x) u .
$$

It is then easy to verify that a function

$$
u \in W^{2, p}\left(0, T ; L^{q}(\Omega)\right) \cap W^{1, p}\left(0, T ; W^{2, q}(\Omega)\right)
$$

is a solution of (1.3) in the sense of the statement if and only if $u$ is a solution of the problem (4.4) from Theorem 4.2. For one implication one should note that $D(\mathcal{B}) \subset L^{p}\left(0, T ; W^{2, q}(\Omega)\right)$, so that $u+\dot{u} \in D(\mathcal{B})$ and $u(0)=0$ implies $u \in W^{1, p}\left(0, T ; W^{2, q}(\Omega)\right)$.

It has been shown in [24, Theorem 4.1] that, under the conditions (4.6)-(4.8), the family $(B(t))_{t \in[0, T]}$ satisfies the Acquistapace-Terreni conditions. To be precise, in [24], the proof is only given for Neumann type boundary conditions, that is, $c^{1}=0$, but the proof for arbitrary positive $c^{1}$, that is, for Robin type boundary conditions, is similar. It is moreover easy to verify that the functions $B_{0}, A_{0}:[0, T] \rightarrow \mathcal{L}\left(W^{1, q}(\Omega), L^{q}(\Omega)\right)$ are bounded and strongly measurable by the assumptions (3.7) and (3.10) (which we assumed with $p=\infty$ ).

By [7, Theorem 6.1], the operators $-B(t)$ generate $C_{0}$-semigroups on $L^{q}(\Omega)$ satisfying Gaussian estimates. The Gaussian estimates are uniform in $t$ due to the uniform boundedness and ellipticity of the coefficients $b_{i j}$. Moreover, as is shown in [7], the first order problem (1.2) is well-posed in $L^{q}(\Omega)$ in the sense that it generates a strongly continuous evolution family. In particular, the closure of $\mathcal{D}+\mathcal{B}$ is the negative generator of a $C_{0}$-evolution semigroup on $L^{p}\left(0, T ; L^{q}(\Omega)\right)$ and hence condition (4.3) holds. By [14, Theorem 1], the first-order problem (1.2) has $L^{p}$-maximal regularity.

Noting finally that the space $W^{1, q}(\Omega)$ is close to $L^{q}(\Omega)$ compared to $D(B(t))$ uniformly in $t$, we deduce the claim from Theorem 4.2.

REMARKS 4.5. (a) In the above proof, the maximal regularity of the first order problem (1.2) can also be shown by using results from [19] or [21].

(b) It is in principle also possible to allow non-zero initial values $u_{0}$ and $u_{1}$ in Corollary 4.4 (compare with the discussion of (3.4) in the constant 
domain case). For this to be applicable, it would be necessary to identify the trace space

$$
\operatorname{Tr}_{p}:=\{(u(0), \dot{u}(0)): u \in D(\widetilde{\mathcal{L}})\} .
$$

We have not attempted to identify this space.

\section{References}

[1] P. Acquistapace and B. Terreni, A unified approach to abstract linear nonautonomous parabolic equations, Rend. Sem. Mat. Univ. Padova 78 (1987), 47-107.

[2] H. Amann, Maximal regularity for nonautonomous evolution equations, Adv. Nonlinear Stud. 4 (2004), 417-430.

[3] W. Arendt, R. Chill, S. Fornaro and C. Poupaud, $L^{p}$-maximal regularity for nonautonomous evolution equations, J. Differential Equations 237 (2007), 1-26.

[4] P. Cannarsa, G. Da Prato and J.-P. Zolésio, The damped wave equation in a moving domain, ibid. 85 (1990), 1-16.

[5] R. Chill and S. Srivastava, $L^{p}$-maximal regularity for second order Cauchy problems, Math. Z. 251 (2005), 751-781.

[6] -,$-L^{p}$ maximal regularity for second order Cauchy problems is independent of $p$, Boll. Un. Mat. Ital. (9) 1 (2008), 147-157.

[7] D. Daners, Heat kernel estimates for operators with boundary conditions, Math. Nachr. 217 (2000), 13-41.

[8] G. Da Prato and P. Grisvard, Sommes d'opérateurs linéaires et équations différentielles opérationnelles, J. Math. Pures Appl. 54 (1975), 305-387.

[9] R. Dautray et J.-L. Lions, Analyse mathématique et calcul numérique pour les sciences et les techniques. Vol. VIII, INSTN: Collection Enseignement, Masson, Paris, 1988.

[10] G. Di Blasio, $L^{p}$-regularity for solutions of nonautonomous parabolic equations in Hilbert spaces, Boll. Un. Mat. Ital. C (6) 1 (1982), 395-407.

[11] - Maximal $L^{p}$ regularity for nonautonomous parabolic equations in extrapolation spaces, J. Evol. Equ. 6 (2006), 229-245.

[12] A. Favini, Parabolicity of second order differential equations in Hilbert space, Semigroup Forum 42 (1991), 303-312.

[13] M. Hieber and S. Monniaux, Pseudo-differential operators and maximal regularity results for non-autonomous parabolic equations, Proc. Amer. Math. Soc. 128 (2000), 1047-1053.

[14] - - - Heat kernels and maximal $L^{p}-L^{q}$ estimates: the nonautonomous case, J. Fourier Anal. Appl. 328 (2000), 467-481.

[15] M. Hieber and J. Prüss, Heat kernels and maximal $L^{p}-L^{q}$ estimates for parabolic evolution equations, Comm. Partial Differential Equations 22 (1997), 1647-1669.

[16] T. Kato and H. Tanabe, On the abstract evolution equations, Osaka Math. J. 14 (1962), 107-133.

[17] A. Lunardi, Analytic Semigroups and Optimal Regularity in Parabolic Problems, Progr. Nonlinear Differential Equations Appl. 16, Birkhäuser, Basel, 1995.

[18] S. Monniaux and J. Prüss, A theorem of Dore-Venni type for noncommuting operators, Trans. Amer. Math. Soc. 349 (1997), 4787-4814.

[19] P. Portal and Ž. Štrkalj, Pseudodifferential operators on Bochner spaces and an application, Math. Z. 253 (2006), 805-819. 
[20] J. Prüss and R. Schnaubelt, Solvability and maximal regularity of parabolic evolution equations with coefficients continuous in time, J. Math. Anal. Appl. 256 (2001), 405-430.

[21] J. Prüss and G. Simonett, $H^{\infty}$-calculus for the sum of non-commuting operators, Trans. Amer. Math. Soc. 359 (2007), 3549-3565.

[22] Ž. Štrkalj, A theorem on products of non-commuting sectorial operators, in: Evolution Equations and Their Applications in Physical and Life Sciences (Bad Herrenalb, 1998), Lecture Notes in Pure and Appl. Math. 215, Dekker, New York, 2001, $175-185$.

[23] H. Tanabe, Equations of Evolution, Monogr. Stud. Math. 6, Pitman, London, 1979.

[24] A. Yagi, Parabolic evolution equations in which the coefficients are generators of infinitely differentiable semigroups II, Funkcial. Ekvac. 33 (1990), 139-150.

St. John's College

Oxford OX1 3JP, Great Britain

E-mail: charles.batty@sjc.ox.ac.uk

Laboratoire de Mathématiques

et Applications de Metz - CNRS

Université Paul Verlaine - Metz

UMR 7122, Bât. A, Île du Saulcy

57045 Metz Cedex 1, France

E-mail: chill@univ-metz.fr
Department of Mathematics Indian Institute of Science Bangalore 560 012, India Current address:

Department of Mathematics University of Delhi, Delhi, India E-mail: sachi_srivastava@yahoo.com

Received July 4, 2007

Revised version June 26, 2008 